\title{
BRAUCHBARE ARTEFAKTE. \\ STATISTIKSOFTWARE FÜR DAS PFLEGE-MANAGEMENT IM SPITAL ALS DAS PRODUKT ETHNOGRAPHISCHER ARBEIT
}

\author{
Christoph Maeder
}

Soziologisches Seminar. Universität St. Gallen

\section{Einleitung}

In folgenden Text wird gezeigt, wie mit einer anwendungsorientierten ethnographischen Arbeit ein Darstellungsproblem der Arbeit einer Berufskategorie im Spital bearbeitet wurde und wie daraus ein Darstellungsproblem - oder möglicherweise eine erweiterte Form der Darstellung innerhalb der Ethnographie - entstanden ist. Das Spezielle an der hier vorgestellten Art ethnographischer Darstellung eines Berufsfeldes in einer komplexen Organisation liegt darin, dass die Ethnographie als Methode gewissermassen ,undercover" eingesetzt wurde. Das Produkt der ethnographischen Arbeit in der Form von Software und dazugehörigen Produkten, wie Handbüchern, Formularen, statistischen Präsentationen usw., wird nicht mehr als Ethnographie im herkömmlichen Sinn erkennbar. Dieser verdeckte Einsatz ethnographischer Expertise wird wenn wir die Deutungskulturen und ihre machtmässige Positionierung im organisationellen Feld kennen - als eine notwendige Strategie verständlich werden.

Im ersten Schritt wird der theoretische Rahmen des Konzepts der organisationellen Repräsentationsarbeit im Spital beschrieben und in einem zweiten Schritt auf die spezifischen Schwierigkeiten der Repräsentation von Arbeit im angepeilten Berufsfeld, der Krankenpflege, eingegangen. Im dritten Schritt wird das in einem Feldforschungsprozess konzipierte, brauchbare Artefakt, ein softwaregestütztes Managementinformationssystem für die Krankenpflege mit dem Namen LEP (Abkürzung für "Leistungserfassung in der Pflege“) vorgestellt und mittels eines materialen Fragments dessen ethnotheoretische Fundierung dargelegt. In einem vierten Schritt schliesslich befassen wir uns mit dieser für die ethnographische Darstellungspraxis doch merkwürdigen Form ethnographischer Repräsentation. 


\section{Das Repräsentationsproblem von Arbeit in der Organisation}

Organisationen werden für ihre Mitglieder zu subjektiven und objektiven Phänomenen der Wirklichkeit, indem in alltäglichen, symbolisch codierten Handlungspraktiken immer wieder kommunikativ verdeutlicht wird, welche „Dinge“ für die Handelnden in diesen sozialen Ordnungen vorkommen und Gültigkei haben. Das Fundament der Organisation wird dabei durch die Interaktionen der Mitglieder in einer ethnomethodologischen Sichtweise als ein „doing organization" (Garfinkel und Sacks, 1976. 130-176) immer wieder hergestellt. Dabei gebrauchte Deutungsschemata und ihre kompetente Pragmatik in der Handlungssituation werden - in Anlehnung an Hall (1997. 15-47) - als kulturelle Repräsentationssysteme bezeichnet, die im Verlauf der Sozialisation zum Organisationsmitglied erlernt werden müssen. Doch so fundamental die interaktive Dimension des sozialen Handelns in Organisationen auch ist, so einsichtig ist es, dass sich die prinzipiell flüchtigen Interaktionen auf Interpretationsmaterial als kommunikative Ressourcen stützen müssen, die in objektivierter Form als Text, Bild und anderes mehr verfügbar sind. Im Rahmen dieses Aufsatzes beschränken wir uns auf diesen speziellen Aspekt der sozialen Repräsentation von Arbeit in der Organisation.

Dem sozialen Zwang der objektivierenden Repräsentationsarbeit in Organisationen unterliegen grundsätzlich alle Angehörigen einer Organisation. Je grösser und arbeitsteiliger Organisationen sind und je mehr unterschiedliche Berufe und Professionen in ihr auftauchen, umso wichtiger werden in der Regel die Praktiken der objektivierenden Repräsentation von Arbeit. Dies triff insbesondere für die von uns untersuchte Organisation vom Typ „Spital“ zu. Nur durch die objektivierende Repräsentation von Arbeit sind längere Kooperations- und Koordinationsketten in solchen arbeitsteiligen Kontexten von personenbezogenen Dienstleistungen überhaupt herstellbar.

Doch das enge, funktionale Argument steht nicht allein. Die Sichtweise wie sie insbesondere in vielen Management - und Organisationslehrbüchern als zentral markiert wird - ist für sich allein genommen nämlich zu stark konsensgeladen. 'Je grösser und komplexer Organisationen werden, desto mehr

1 Hier wird inshesondere an die Literatur des Controllings erinnert. in der immer wiede ausgeführt wird. dass Kennzahlensysteme zur Organisationssteuenung erstens nicht nur monetär Grössen umfassen sollen und dass zweitens die umfassende und sachgerechte Dokumentation gewissermassen das Herzstuck erfolgreicher Arbeit sein muss. Nur durch die konstante Verschriftlichung sind ja letztlich all die vorgeschlagenen Berechnungen möglich und wird ein Weg von der Managementstrategie zur Handlung der Mitglieder in der Organisation überhaupt vorzeigbar. Exemplarısch für diese Argumentations- und Herangehensweise steh das Konzept der ...Balanced Score Card "von Kaplan und Norton (1997), das sich gegenwärtig im manageriellen Umfeld schnell verbreitet steigt - soziologisch gesprochen - auch der Bedarf an Legitimationsressourcen zur Durchsetzung von Interessen im innerorganisatorischen, mikropolitischen Aushandlungsspiel über die Wirklichkeit. Dies deshalb, weil Organisationen in Anlehnung an die medizinsoziologischen Arbeiten von Anselm Strauss und seiner Mitarbeitenden als „negotiated orders“ (Aushandlungsordnungen) begriffen (Strauss 1978; Strauss; Schatzman; Ehrlich. Bucher und Sabshin 1963 147-169) - sich in einem permanenten Prozess der Aushandlung zwischen den unterschiedlichen Berufsgruppen und Professionen befinden, ja befinden müssen. Das Argument wird einsichtig, wenn wir bedenken, dass Spitäler über keine einfachen, von allen Zuständigen und Betroffenen anerkannte, normativ hoch gesättigten und damit selbstverständlichen Erfolgskriterien verfügen. So zeigte Strauss in der bereits zitierten Arbeit, dass die generelle ,professional ideology" im von ihm untersuchten Krankenhaus sich einzig auf die gemeinsame Idee „Wir entlassen die Patienten in einem besseren Zustand“ (Strauss et. al., 1963, 148 f.) beziehen liess. Was dieses ,,besser“ für Bedeutungsdimensionen tragen soll, und wie es in concreto - z. B. bei Vorliegen einer chronischen Krankheit - zu verstehen ist, das ist der Stoff, aus dem - je nach Berufsgruppe und wissenschaftlicher Orientierung der Akteure - die Aushandlungsgrundlagen gewoben werden. Während im Gegensatz dazu rein auf wirtschaftlichen Erfolg ausgerichtete Organisationen wenigstens einmal im Jahr über Bilanz und Erfolgsrechnung ihren Erfolg und die Legitimation ihrer Existenz vergleichsweise problemlos begründen können, reicht dieser auch für ein Spital zweifellos wichtige, ökonomische Ausweis eben - entgegen allen Kostendiskussionen im Gesundheitswesen - keineswegs aus. ${ }^{2}$ Hier geht es - neben den stets umstrittenen Ärzteeinkommen - auch um moralische und ethische Diskurse innerhalb der, und über die Medizin. Und solche im Diskursformat nachweisbaren Aushandlungsordnungen sind eben vielschichtiger angelegt, weniger eindeutig zurechenbar in ihren Wirkungslinien, und ausserdem auch von betroffenen Laien als Patienten und Angehörige mitgestaltbar und mitgestaltet.

2 An diesem Punkt scheitern ja in aller Regel auch die gesundheitsökonomischen Rezepturen. Indem immer so getan wird, als sei alles und jedes. das im Spital von Bedeutung ist, über wirtschaftliche Anreizsysteme seuerbar, wird syster. das im Spital von Bedeutung ist, über Zeckrationalität im Sinne Webers (Weber 1972,19 ) aubersehen, dass Im Krankenhaus Gültigkeit erlang. Normative, derökonomischen Gedankenwelt anders als über Geldmittel Gültigkest erlangt. Normat1ve. der ökonomischen Gedankenwelt entgegenstehende Ansprüche sind in grosser Zahl in die moralischen und ethischen Wertstrukturen der Akteursgruppen (Arzte, Pflegende, Sozialarbeitende, Pfarrdienste usw.) eingelagert. So werden beispielsweise in der Schweiz qua gesetzlicher Regelung auch Personen behandelt. die nicht selber bezahlen können, es werden - Singer zum Trotz - auch Leben erhalten, die aus utilitaristischer Sicht nicht mehr sein sollten usw. Was die sogenannten .,inputs“, ,outcomes“ und .outputs“ eines Spitals sind, bedarf im Rahmen dieser Betrachtungsfigur insbesondere auf der Ergebnisseite eines enormem Experteneinsatzes. und sie bleiben trotzdem irreparabel unterbestimmt. 
2.I Unterschiedliche Formen der professionellen Repräsentation der Arbeit im Spitals

Im Rahmen unserer Feldarbeiten sind wir relativ rasch auf nach drei Berufs gruppen geordnete Repräsentationssysteme und deren jeweils in spezifischen Formaten geordnete Objektivationsformen gestossen: die Ökonomie, die ärztliche Medizin und die Pflege. Wenn es auch für einen geregelten Spitalbetrieb noch vieler anderer Berufsgruppen bedarf, so bildet diese Dreiheit doch den definitorischen Kernbereich dieser Betriebskultur ${ }^{3}$ Die Trias findet sich in der Schweizerischen Spitallandschaft fast immer als Führungsstruktur in der Aufbauorganisation in Form von Organigrammen und Funktionsbeschreibungen: An der Spitze der grösseren Spitalorganisationen stehen in der Regel eine auf Zeit vom Ärztekollegium bestimmte ärztliche Direktion sowie die auf unbestimmte Zeit gewählte Verwaltungsdirektion und die Pflegedirektion (auch als Pflegedienstleitung bezeichnet). Umgangssprachlich wird diese Organisationsstruktur als das sogenannte „Dreibein“ bezeichnet, der die Spitalführung insgesamt obliegen soll.

Trotz formaler Gleichstellung der drei Disziplinen in der normativen Vorstellung über die Spitalführung fällt bei genauerer Betrachtung auf, wie die objektivierenden Repräsentationen und der Grad ihrer klaren Deutbarkeit stark zu Ungunsten der Krankenpflege ausfallen. Während - stark vereinfacht ausgedrückt - die Ärzteschaft die Codierung „krank“ und ,gesund“, ,gelungen“ und „,nicht gelungen“, ,normal“ und ,abweichend“ konstant in ihre Dokumentationen und Unterlagen einarbeitet, verfügt die ökonomisch zuständige Verwaltung über den Apparat der Kostenrechnung und daraus abgeleiteter Systematiken der Darstellung von Erfolg oder Nichterfolg in der Arbeit. In ihren Bemühungen zur erfolgreichen Praxis werden Medizin und Ökonomie zudem von universitär gestütztem Orientierungs- und Handlungswissen zusätzlich von aussen alimentiert und legitimiert. Beide Objektivierungsspraktiken und deren Systematik sind im Format der Zahl codiert oder einfach codierbar Die zumeist naturwissenschaftlich-biologisch hervorragend, aber sozialwissenschaftlich kaum ausgebildete Ärzteschaft orientiert sich denn auch in der Darstellung medizinischer Praxis mehrheitlich am ersten Modell. Folgerichtig gilt auch die sogenannte Doppelblindstudie in aller Regel als die Vorlage für Wissenschaftlichkeit in der Medizin überhaupt ${ }^{4}$

3 In der Regel arbeiten in einem Spital Angehörige mehrerer Dutzend Berufe, und auch auf de Professionsebene sind neben dem medizinischen Personal immer auch noch Angehörige der juristischen, der psychologischen, der theologischen und weiterer Professionen tätig.

4 Zur Tragweite dieses Wissenschaftsmodells im medizinischen Bereich, insbesondere in der naturwissenschaftlich schlecht erfassbaren Sozialpsychiatrie, siehe Finzen. 1999. 116-144.
Die Krankenpflege verfügt - abgesehen von in die ärztlichen Dokumentationen integrierten, sogenannten Pflegedokumentationen - über keine tatsächlich ihrer eigenen und exklusiven Deutungsmacht unterstehende Form der Objektivation ihrer Arbeit. Und sie hat selber auch keine eigenen, für Dritte kommunizierbare, ja gar von wissenschaftlicher Expertise unterstützte Theorien darüber. Dies bedeutet nicht - um Missverständnissen vorzubeugen - dass es nicht zahlreiche sogenannte „Pflegephilosopien“ oder Pflegediagnosesysteme (z. B. Doenges und Moorhouse, 1993) gibt. Nur kommen diese bisher praktisch ausschliesslich aus dem angelsächsischen Raum, wo die Akademisierung des Berufsfeldes schon weiter fortgeschritten ist. Zudem liegen meistens in sich konkurrierende Fassungen unterschiedlicher Forschergruppen vor, die, soweit bekannt, nie je wirklich umfassend und in nennenswertem Umfang in die lokale Praxis integriert worden sind. Diese Konzeptionen sind allesamt und sonders kulturfremd für die Pflegepraxis in der Schweiz und andererseits häufig derart komplex (siehe z. B. RAI/RUG (James, 1997); NIC, eine Einheitssprache in der US-amerikanischen Pflegediagnostik (McCloskey und Bulechek. 1986)), dass sie sich als Instrumente des tagtäglichen Gebrauchs auch im Management der Pflege von selber verbieten. Als kommunikative Ressourcen stehen diese Instrumente eher für die Selbstinszenierung an Kongressen und Tagungen zur Anmeldung des Anspruchs auf Wissenschaftlichkeit zu Verfügung, als für die alltägliche Organisation der Arbeit und die Durchsetzung von Interessen innerhalb der Organisation.

Wie festgestellt wurde, dokumentieren die beiden einflussreichen Professionen im Spital - Ärzteschaft und Verwaltung - ihre wichtigen, für die Verhandlungsordnung entscheidenden Darstellungssysteme letztlich zahlenförmig: Eingriffs- und Operationsstatistiken mit abgeleiteten weiteren Darstellungen und Analysen sowie buchhalterisch-kostenmässige Darstellungsformen bilden die Schlüsseldaten der aggregierten Rekonstruktionen vergangener Zeitperioden der Arbeit im Spital und im Gesundheitswesen überhaupt. Mit Umberto Eco gesprochen, folgen Ärzte und Verwalter damit einem ausgezeichneten und erst in modernen Gesellschaften generalisierten Weg der Herstellung von Wissen über die Welt. Im Buch das Foucault'sche Pendel lässt Eco einen seiner Protagonisten sagen, dass das Universum eben doch und entgegen aller Kritik ein wunderbares Konzert von Zahlenkorrespondenzen sei und dass die Lektüre der Zahl und ihre symbolische Deutung ein privilegierter Weg zur Erkenntnis darstellten (Eco, 1993, 338 f.).

Im Gegensatz dazu war, bis zur Entwicklung von LEP, die Pflege auf Zahlen anderer angewiesen, und es blieb ihr demzufolge der ausgezeichnete Weg der Erkenntnis auch über ihre eigene Welt versperrt. Sie konnte als sogenannte „Semi-Profession“ (Etzioni, 1969) nur wenig auf alltäglich brauchbares 
Wissen mit universitärer Legitimation oder wenigstens managerieller Herkunft zurückgreifen. Dieser Mangel an einer Datenbasis in Zahlenform über das eigene berufliche Tun hat in der Vergangenheit einerseits zur Etablierung einer Abhängigkeit von Daten der Verwaltung geführt und andererseits verhindert, dass die Pflege - abgesehen von Sonntagsreden - überhaupt als produktiver Faktor in Berichten über vergangene Zeitperioden der Arbeit ausgewiesen werden musste. Zumeist erscheinen Angaben über die Pflege auch heute noch fast ausschliesslich auf der Ausgaben- und Kostenseite. Eigene Leistungen werden bestenfalls in der Form von sogenannten Pflegetagen ausgewiesen. Wobei allerdings der sogenannte Pflegetag lediglich die datumsmässig geordnete Anzahl vorhandener Personen mit Patientenstatus in einer Organisation angibt und keine Angaben über die Pflegearbeit im engeren Sinn machen kann. Ein Blick in ein paar zufällig ausgewählte Jahresberichte von Spitälern wird diese Ausführungen der interessierten Leserschaft bestätigen. Erst in jüngster Zeit beginnen sich in der Schweiz Bemühungen über eine weiterreichende Fassung der Pflege in Zahlenform abzuzeichnen, wie z. B. das Projekt „Nursing Data“ belegt (Berthou und Junger, 1999).

Im Vergleich zu den elaborierten Repräsentationspraktiken im ärztlichen Sektor (Diagnosecodierungen, bildgebende Verfahren aller Art, Beschreibungen von Eingriffsarten, Erfolgsquoten, Laborwerte usw.) und in der Verwaltung (die gesamten komplexen Darstellungsformen der Kostenrechnung, der Buchhaltung. der Personalführung usw.) konnte und kann die Pflege bis heute nicht mithalten. Sie war und ist deshalb als weiblich dominierte Halbprofession ständig in Gefahr, von der eigenen Führungsspitze, aber insbesondere von den anderen professionell organisierten Berufen im Spital als eine nicht-rationale Klagekultur typisiert zu werden. Und sobald die Pflegenden sich in moderneren Formen der Teamarbeit mit dem dadurch bekanntermassen höheren Aufwand an Besprechung und Koordinationsarbeit (Stichworte sind „Primary Nursing" oder die Bezugspersonenpflege im Team) zu organisieren beginnen, kursiert bei den anderen im Betrieb schnell das Vorurteil: ,die reden ja nur, statt dass sie arbeiten".

Die gegenseitige Stereotypisierung von Ärtten und Pflegenden folgt in aller Regel, soweit wir beobachten konnten, einem klaren Muster: Während die Pflegenden vermuten und sich darüber auch beklagen, die Ärzte würden sich mehr für die Zahlen (Laborwerte. Medikamentendosierungen, wissenschaftliche Messreihen. Anzahl von Operationen usw.) über „Fälle“, als für die Patientinnen und Patienten ,,in ihrer Ganzheit" interessieren, so trauen die Ärztinnen und Ärzte in der Regel den Pflegenden im Umgang mit deren eigener Arbeit häufig wenig zu. Sie unterstellen oftmals subtil, die Pflegenden seien im Schwatzen besser als bei der eigentlich sinnvollerweise zu leistenden
Arbeit. Dass diese Stereotypisierungen selten direkt und offen ausgetauscht werden, versteht sich angesichts ihres diffamierenden Potentials und der von Dritten an beide Berufsgattungen gehegten Erwartungen von selbst. Für die ökonomisch Befassten schliesslich ist und bleibt die Pflege im Spital ein Buch mit sieben Siegeln, das sie in aller Regel analog der Vorurteilsstruktur der Ärzte beschreiben und deuten.

Zusammenfassend können wir festhalten, dass die Dokumentationspraktiken als Repräsentation der Arbeit im Spital stark professionsgebunden und bei Arzten und in der Verwaltung in erster Linie an Zahlen gebunden ist. Die Pflege steht im offenen Gegensatz dazu. In der Regel verfügt sie über keine ausschliesslich in ihrer Definitionsmacht stehenden Konzepte der Repräsentation ihrer Arbeit. Diese Nichtrepräsentation der Pflegearbeit hat nun zweifellos Konsequenzen: die Pflege läuft stets Gefahr, als nicht fassbar und somit auch nicht als eine rationale Handreichungspraxis zu gelten. ${ }^{5}$ Damit einher geht sehr überspitzt formuliert - eine geschlechtsgebundene Typisierung in männlich-wissenschaftliche Repräsentation im Zahlenformat und eine weiblich-klageartige im Schwatzformat. Diese unterschiedlichen Deutungskulturen der organisationellen Wirklichkeit führen - über hier nicht näher beschriebene Wirkungslinien - zu einer hierarchisch klaren Unterordnung der Pflege im Spital insgesamt. Das Regime des ärztlichen Blicks - um mit Foucault (1988) zu sprechen lässt es bei der Selbstbeschreibung der Arbeit nicht zu, dass in diesem Kontext bei der Objektivierung der geleisteten Arbeit mit anderen Darstellungsformen als mit Zahlen operiert wird. Wenn die mächtigste Profession in der modernen Gesellschaft sich auf Zahlen verlässt, dann ist es einsichtig. dass damit Wirklich-

Aus diesem Grund vermuten wir übrigens, dass auch das häufig anzutreffende Phänomen der Arbeitszeiterfassung und -kontrolle nur im Pflege- und Verwaltungsbereich aber - abgesehen von punktuellen Studien im Umfeld politischer Konfliktfälle - kaum je bei den Arzten anzutreffen sind. Oder anders formuliert: Bei den Ärzten gelten andere und insbesondere von der eigenen Profession kontrollierte Erfolgskriterien. Für die Assistenz- und Unterassistenze von hat dies - infolge ihrer inferioren Einflussmöglichkeiten im paternalistisch - fötfeusistenzkrafte Führungs- und Organisationsmodell, das für Ärzte in der Schweiz prakpatfeudal geprägten prozentig gilt - durchaus nicht nur positive Konseguen Arbeitszeiten - je nach Standpunkt und Untersuchungenzen. Dass ihre zweifellos sehr langen - als normal gelten, verdanken sie auch dieser Profesionsman $48 \mathrm{~h}$ und $60 \mathrm{~h}$ pro Woche Höherstehenden selbstverständlich sesser auch dieser Professionsmacht. welche die sozial Erklärung des Phänomens der langen Arbeitszeiten von Assistenzenden. Diese soziologische Erklärung des Phänomens der langen Arbeitszeiten von Assistenzärzten und -ärztinnen im sind ökonomischer (zu wer Organisation selber ein Tabu dar. Die akzeptierten Erklärungen sind ökonomischer (zu wenig Stellen. zu wenig Geld) und ethisch-moralischer Art (wir tun alles für die Patienten). Interessant ist dabei, dass sich dieses Phänomen nicht nur in der Schweiz finden lässt, wie die wohl berühmteste Untersuchung zur Sozialisation von Ärzten. bereits 1961 veröffentlicht zeigt. Howard S. Becker und seine Mitarbeitenden (Becker et. al. 1997. 239-273) beschreiben am Beispiel von amerikanischen Assistenzärzten, wie autoritäre We nachgerade typisch sind für den Werdegang von Medizinern. 
keit definiert wird und sich andere unterzuordnen haben. Es ist demnach folgerichtig zu erwarten, dass in einer solchen modernen, multiprofessionell bestückten Organisation mit den dominierenden Deutungskulturen der Ärzte und der Ökonomen Repräsentationsformen ohne Zahlenformat wenig Chancen haben.

\section{Angewandte Ethnographie: Geschichte und Konstruktion von LEP}

In die im letzten Kapitel beschriebene Repräsentationskonstellation hinein haben wir den Auftrag bekommen, die Pflegearbeit zu objektivieren und diese Objektivationen als Führungsinstrumente brauchbar zu machen. Es ging darum, ein Messinstrument für die Pflegearbeit zu entwickeln. das in ein Computerprogramm umgesetzt werden sollte. Derartige Produkte werden im medizinisch-manageriellen Kontext „Leistungserfassungsinstrumente“ oder ,Patientenklassifikationssysteme" genannt und sind im Bereich der ärztlich-ökonomischen Zuständigkeiten in grosser Zahl verfügbar: Von den in der Konstruktion undurchsichtigen DRG's (= diagnose related groups) aus den USA über die Klassifikationen von Krankheiten im ICD-Format (= International Classification of Diseases mit den Varianten des Katalogs Nr. 9 und Nr. 10) bis hin zu den neuesten Kategoriensystemen der WHO mit der Abkürzung ICIDH zur Funktionsfähigkeit und Behinderung (Assessment. Classification and Epidemiology Group, 2000) und den medizinischen Tarifsystem der Ärzte namens TARMED spannt sich ein weiter Bogen von Beschreibungsmöglichkeiten von Patientinnen und Patienten sowie von den ihnen zukommenden Leistungen.

\section{I Spezifika der Pflegearbeit im Spital im Hinblick auf deren Verrechnung}

Die ..Verrechnung" der Pflegearbeit, d. h. deren zahlenmässig sinnvolle Beschreibung, stösst sehr schnell an Grenzen. Um Tätigkeiten messen zu können, müssen sie analytisch und empirisch klar voneinander trennbar sein. Das ist jedoch in der Pflegearbeit als einer personenbezogenen. interaktiv erbrachten Dienstleistung oft nicht der Fall. Da Produktion und Konsum solcher Dienstleistungen gemäss dem .,uno-actu Prinzip" vielfach zusammenfallen, lässt sich auch bei genauer Beobachtung kaum feststellen, was wann wo beginnt, was parallel ausgeführt wird, und wann etwas endet. Es ist nur analytisch und um den Preis einer groben, ja fast brachialen Vereinfachung möglich, sich für eine klare Zuschreibung zu entscheiden. Wir stossen hier auf ein schwieriges Messproblem, es ist aber nicht das einzige Problem.
Verschiedene Bereiche der Pflegearbeit lassen sich kaum sinnvoll oder überhaupt nicht quantitativ erfassen. Dazu gehören eine Reihe von Arbeiten, die häufig übersehen werden, jedoch - wie einschlägige medizinsoziologische Arbeiten (Strauss et. al.. 1975, 175-202) gezeigt haben - für den Spitalbetrieb insgesamt von zentraler Bedeutung sind. In erster Linie sind es Handlungen, die auf die Sicherheit, das Wohlbefinden oder die Gefühlslage von Patienten und Patientinnen abzielen. Strauss und seine Mitarbeitenden haben diese als Sicherheitsarbeit (,,safety work“), Behaglichkeits- und Trostarbeit (,comfor work") und Gefühlsarbeit (,,sentimental work") bezeichnet. 'All diese Arbeiten werden zur Hauptsache von Pflegepersonen verrichtet. Da es sich vorwiegend um qualitativ-beziehungsmässige Aspekte der Pflege handelt, können diese mit einem quantitativen Messinstrument wenig oder gar nicht erfasst werden.

Weitere für die Pflegenden wichtige Aspekte fehlen in jedem Leistungserfassungsinstrument oder in quantifizierten Darstellungen notwendigerweise vollständig. Ihre Arbeit und ihre Berufsrollen sind von einer erheblichen sozialen Vielschichtigkeit und Vieldeutigkeit. Nicht erwähnt ist ausserdem, dass diese Arbeit mit berufsspezifischen Eigenheiten belastet ist, wie man sie wahrscheinlich nur noch bei wenigen anderen Berufen findet. Faktoren wie Verantwortungsdruck gegenüber Ärzten, Patienten und Angehörigen, die Konfrontation mit Krankheit und Tod, die oftmals nötige Durchbrechung sozialer Tabus, wie z. B. Nacktheit, Scham und Abhängigkeit, und die Fragen der Berufsidentität können mit keiner derartigen Methode erfasst werden und sind dennoch dem Kern der Arbeit zuzurechnen (siehe dazu Schneider, 1987, 7-24).

\subsection{Das Instrument}

Trotzdem haben wir uns Anfang der Neunzigerjahre trotzdem entschlossen, ein quantifizierendes Managementinstrument für die Pflege zu entwerfen, um eine objektivierte Darstellung dieser Arbeit zu haben und sie so steuerbarer und für Dritte kommunikativ anschlussfähig zu machen. Die klassische Feld arbeit für diese Untersuchung wurde zwischen 1989 und 1992 im Kantons spital St. Gallen und im Universitätsspital Zürich intensiv begonnen und seither nur sporadisch weitergeführt.? Die mit den Verfahren der ethnographischen

6 Gefühtsarbeiten sind in den letzten zwei Jahrzehnten vermehrt in den Aufmerksamkeitshorizont der Organisationsforschung gekommen. Eine Einführung findet sich bei Fineman (1993, 9-35). Der Klassiker unter diesen Studien ist die Arbeit von Russel-Hochschild (1983) über die Gefühlsarbeit von Flight Attendants und Studierenden.

7 An dieser Stelle ist denjenigen zu danken. die das Projekt überhaupt erst möglich gemacht haben. Dies sind insbesondere Josef Arnold (Leiter Pflegedienst am Universitätsspitäzürech) und Ruedi Mouron (Leiter Pflegedienst am Kantonsspital St Gallen). Neben diesen beiden unermüdlichen Förderern kommen auch Ulrich Bamert, heute Geschät 
Semantik (Maeder und Brosziewski, 1997, 335-362) gewonnenen Kategoriensysteme zur Arbeitspraxis in der Krankenpflege im Spital konnten jedoch vor dem Hintergrund der dominierenden Deutungskulturen naturwissenschaftlichmanagerieller Art nie als ethnographische Befunde ausgewiesen und als eine Ethnographie dargestellt werden, da ja das explizite Ziel des Auftrags darin lag. ein Zahlensystem für die Pflegenden zu konstruieren. ${ }^{8}$ Die erste Modellierung der Pflegearbeit in dieser speziellen und ungewohnten Zahlenform erschien in als Buch (Güntert und Maeder, 1994). wurde anschliessend verfeinert und den sich in den Neunziger Jahren wandelnden Gegebenheiten der Spitalorganisation immer wieder angepasst (Maeder, Brügger und Bamert 1998. 1999a. b). ${ }^{9}$ In ethnographischer Selbstbeschreibung ausgedrückt, haben wir ein brauchbares Artefakt für die Auftraggeber hergestellt.

Gemäss managerieller Sprachregelung trägt das Kind eine modische Abkürzung. heisst heute LEPTM und ist eine registrierte Marke. Eine professionell geführte Geschäftsstelle mit drei festangestellten Mitarbeitenden betreut den Verkauf und die Wartung der in der Schweiz am weitesten verbreiteten Methode dieses Typs. LEP ist inzwischen von fünf verschiedenen Softwarefirmen programmiert worden und wird mittlerweile in mehr als 70 schweizerischen Spitälern routinemässig eingesetzt. Gegen Ende 2000 stehen gar Pilotläufe mit der Methode auf Intensivstationen in Deutschland an. Möglicherweise wird damit der bisher nationale Charakter von LEP gesprengt. LEP hat sich in der Praxis als eine sehr brauchbare Art der Darstellung von Pflegearbeit bewährt und gehört mittlerweile selber zur Arbeitskultur der Pflege im Spital in der

grosse Verdienste zu für seinen engagienten Einsatz zugunsten der Verbreitung des Instruments. der Universitäı St Gallen und Mitstreiter in Sachen LEP im Feld danke ich für die zahlreichen Diskussionen und Hinweise auf der langen Reise zu einer brauchbaren Pflegestatistik.

Weniger Verständnis für die Anliegen der Pflege hat in den leizten Jahren die Gesundheitsdirektion des Kantons Zürichs gezeigt. Obwohl die Methode von den Pflegenden im Kanton Zürich massgeblich mitgeprägt und mitentwickelt worden ist und die Pflege erstklassige Daten für die Steuerung erzeugt. hat die Gesundheitsdirektion bis heute kein produktives Interesse an der Methode gezeigt. Damit wurden und werden selbstverständlich viele motivierte Personen in der Pflege im Einflussbereich dieser Behörde unnötig vor den Kopf gestossen.

8 Für eine vollständige, theoretische und methodologische Fundierung der hier verfolgten Empırie wird verwiesen auf die beiden Bände zur Ethnographie in der Traditionslinie der .Ethnoscience" von Werner und Schoepfle (1986, 1987).

9 Für diese Konstruktionsarbeit war sicher hilfreich. dass der Autor dieses Textes von 1974-77 die Berufsausbildung zum diplomienen Krankenpfleger durchlief und zwischen 1978 und $1983 \mathrm{int}$ Spital in dieser Funktion gearbeitet hat. Insbesondere die Vertrautheit mit den in Feld verwendeten Begriffen und deren Deutung machte es möglich, einen an den Relevanzen der tatsächlichen Berufspraxis ausgerichteten Katalog von pflegerelevanten Tätigkeitskategorien (mit)herzusiellen. Diese Mitgliedschaft im Feld war insgesamt sicher von Vorteil, doch ist sie keine Voraussetzung. un solche Arbeiten durchführen zu können: Genaues Beobachten und Hinhören und die Beherrschung des ethnographischen Handwerks genügen deutschsprachigen Schweiz. ${ }^{10}$ Wie fest das Instrument bereits in der Pflege verankert ist, illustriert der folgende Vorfall. Als in einem Warnstreik die Pflegenden in verschiedenen Zürcher Spitälern anfangs des Jahres 2000 auf alle administrativen Tätigkeiten verzichteten, wurde LEP vom „Bleistiftstreik“ ausgenommen und trotz seines zweifellos administrativen Charakters weitergeführt.

Das Kernstück von LEP besteht in der Anwendung aus einem Variablenkatalog von 80 Tätigkeitskategorien. Eine Variable steht für eine bestimmte pflegerische Tätigkeit, die einem Patienten oder einer Patientin direkt zugeordnet werden kann. Jede einzelne Pflegevariable umfasst eine Bezeichnung. eine Definition, illustrative Beispiele, eine Anwendungsanleitung und einen festen Zeitwert. Der Zeitwert ist so ausgelegt, dass eine ausgebildete und berufserfahrene Pflegeperson im Durchschnitt eine solche pflegerische Tätigkeit unter Einhaltung der qualitativen Standards des Berufes ausführen kann. Die Zeitwerte sind als normative Vorgaben konzjpiert und absichtlich in der exklusiven Definitionsmacht der Pflege verankert. Sie wurden und werden von einem Expertengremium unter Zuhilfenahme von periodisch durchgeführten Arbeitszeitmessungen festgelegt.

Einmal täglich erhebt das Pflegepersonal pro Patient oder Patientin, welche Variablen wie häufig auftraten. Die Erfassung kann dabei mittels Barcode. optischem Belegleser, PDA (Personal Digital Assistant) oder auch ,online“ erfolgen. Diese sogenannten „Patientendaten“ über die applizierte Pflege bilden die eine Seite des Instruments. Die andere Seite bilden die Arbeitszeiten des Personals, die ebenfalls berechnet werden. Diese zwei Datenkategorien stellen die Grundlage für zahlreiche Auswertungen dar, die nun möglich sind. Solche Auswertungen dienen den Vorgesetzten zur Pflege der kurzfristigen Steuerung ihres Verantwortungsbereiches und den oberen Pflegekadern für die langfristige Planung. Das Produkt lässt sich auf den ersten Blick von einem traditionell hergestellten, quantitativen Messinstrument nicht unterscheiden. Einen Überblick über das Instrument findet die interessierte Leserschaft auf der Website www.lep.ch.

10 Die Methode konnte bisher trotz einer französischsen Fassung in der Westschweiz nicht verbreitet werden. Eine auf der gesundheitspolitischen Ebene äusserst aktiv lobbyierende Gruppierung um ein Lausanner Gesundheitsinstitut hat dies bisher unter Rückgriff auf ein Franko-Kanadisches Modell bisher verhindert. Allerdings können wir uns des Eindrucks nicht erwehren, dass dieses. Modell, welches den vermuteten Bedarf und nicht die abgegebene Pflege abbildet, nicht so erfolgreich implementier ist wie LEP. Unseres Wissens setzten einzig die beiden Universitätsspitaler in Genf und Lausanne die wesentlich teurere Methode regelmässig ein, und dies nicht einmal in allen Betrieben. Anfragen für eine Liste von Anwenderbetrieben und für Daten zu Vergleichsz.wecken wurden bisher jedenfalls inmer wieder worreich
verhinder. 
3.3 Sprache als Träger von Berufswissen: Soziolinguistisches Fundament einer Statistik

Das Aussergewöhnliche des Instruments sehen wir nicht so sehr in seiner Endform, als vielmehr in seinem Konstruktionsprozess. Die Grundlage der Entwicklung dieses Instruments bilden Techniken der qualitativen Sozialforschung, und zwar die der teilnehmenden Beobachtung mit Pflegenden auf den Stationen und Gruppengespräche mit weiteren sachverständigen Personen aus der Pflege, wie z. B. Pflege-Expertinnen mit Höherer Fachausbildung, Oberschwestern, Leitungen Pflegedienst usw. Als besonders fruchtbar hat sich dabei die Form des Gruppengesprächs mit den Pflegenden herausgestellt. Unseres Erachtens dürfte ein Teil des Erfolges dieses Zugriffs auf die organisationelle Wirklichkeit damit zusammenhängen, dass diese Gesprächsformen auch sonst im kulturellen Bezeichnungsrepertoire der Organisation verankert sind, als Rapporte und Teamsitzungen. An Rapporten, denen Funktionen wie Arbeitsplanung, Arbeitsvergabe, Arbeitskoordination und Arbeitskontrolle zukommen, sind die Pflegenden stets damit beschäftigt, Beschreibungen ihrer Arbeit zu kommunizieren. Die Pflege wird so gewissermassen „,natürlich“ und für den Ethnographen zugänglich in Sprachkategorien gefasst, die auf die dazugehörigen beruflichen Wissensbestände verweisen. Der Soziolinguist George Lakoff beschreibt die Bedeutung solcher Sprachkategorien folgendermassen:

There is nothing more basic than categorization to our thought, perception, action, and speech. Every time we see something as a kind of thing, for example, a tree, we are categorizing. Whenever we reason about kinds of things - chairs, nations, illnesses, emotions, any kind of thing at all - we are employing categories. Whenever we intentionally perform any kind of action, say something as mundane as writing with a pencil, hammering with a hammer, or ironing clothes, we are using categories. The particular action we perform on that occasion is a kind of motor activity (e.g. writing, hammering, ironing), that is, it is in a particular category of motor actions. They are never done in exactly the same way, yet despite the differences in particular movements, they are all movements of a kind, and we know how to make movements of that kind.

Lakoff, 1990, 5 f.

Im ersten Zugriff wurde denn auch nichts anderes hergestellt als ein Lexikon der praktischen Tätigkeiten in der Sprache des Feldes. Dieses rekonstruierte Lexikon als lebensweltliches Konstrukt erster Ordnung (vgl. Eberle, 1984, 81147) besteht zumindest in der Schweiz - anderenorts mögen andere Arbeitskategorien in den Zuständigkeitsbereich der Pflegenden fallen - je nach Tätigkeitsbereich aus zwölf oder dreizehn Domänen in der Bezeichnung der Tradition der ethnographischen Semantik (vgl. Maeder \& Brosziewski. 1997, 335-
362). Diese Domänen sind alle mit der Relation ,.ist eine Art Arbeit in der Pflege" mit ihren semantischen Komplementen feinerer Abstufung verbunden. Die Komplemente ihrerseits tragen Attributdimensionen der Aufwendigkei von „,selbständig“ bis „,sehr aufwendig“ im Bereich der sogenannten ..Grundpflege" oder sind im Bereich von sogenannter Behandlungspflege nach den Anspruchsgraden aus Sicht der Pflegenden konnotiert. Die Gewinnung einer solchen Domäne soll nun anhand eines materialen Fragments exemplarisch dargestellt werden. Anlässlich eines Rapports konnte folgendes Gespräch verfolgt werden:

Schwester: Hat schon jemand bei Frau Meier die Körperpflege ge macht?

Pfleger: Anna wollte das noch vor dem Kaffee machen, doch dann ist sie zu mir gekommen und wollte, dass jemand Diplomierter das macht. Frau Meier geht es schlecht, sie ist jetzt sehr aufwendig. Das kann Anna nicht mehr machen mit den vielen Redons und den anderen Schläuchen.

Schwester: Okay, dann gehe ich zu ihr und mache die Ganzwäsche in Bett. Aber du musst mir helfen, denn sie braucht auch noch einen Verbandwechsel. Den kann ich nicht alleine machen, und wir sollten sie nicht zuviel stören. Wir machen alles zusammen, den Redonwechsel, den einfachen Verband und die Infusionen. Ich bereite das vor.

Pfleger: Wer macht jetzt genau was?

Schwester: Ich mache dann die Ganzwäsche, und du kannst ja den Verband und das andere Zeug vorbereiten. Du kommst einfach nach zehn Minuten hinzu mit dem Verbandwagen und dem Rest und wir schauen dann.

Pfleger: Dann kann aber Anna dafiur Herrn Müller machen, der braucht nur etwas Hilfe beim Aufstehen.

In diesem beispielhaft dargelegten Gespräch werden verschiedene Konzepte aus der Arbeit in der Pflege ausgetauscht. Es geht darum herauszufinden, wie und 'om wem die Körperpflege einer Patientin durchgeführt werden soll. Die ser Begriff „Körperpflege“ bildet hier den Oberbegriff einer Domäne oder eines semantischen Feldes, dem in der Pflege zentrale Bedeutung zukommt, da über die mit diesem Konzept verbundenen komplementären Kategorien ein feines und implizit in der Berufskultur hoch standardisiertes Raster zur Arbei am Patientenkörper versprachlicht wird. Die Beteiligten stellen zusätzlich fest dass es sich um eine „Ganzwäsche im Bett“" handelt, was angesichts der zusätzlichen Erschwernisse durch „Schläuche“ denn auch automatisch als anspruchsvolle Arbeit gilt, für die es spezielle Kenntnisse braucht. Die Schwesternhilfe 
Anna traut sich dies alles wegen der vielen zu- und abführenden Leitungen zur Patientin (Redons sind spezielle Wunddrainagen, Infusionen sind zuführende Leitungen in das venöse Blutsystem) nicht $z u$, und sie hat dementsprechend Leitungen in das venose Bluts pflege angelagerten Tätigkeit des geplanten Verbandwechsels, der seinerseits pflege angelagerten Tatigkeit des geplanten Verbandwechsels, der seinerseits einer anderen Domäne mit dem Oberbegriff „Behandlung “ angehört und in die Attributdimensionen ,,einfach“, ,,aufwendig" und ,sehr aufwendig“ eingeteilt ist, wird sichtbar, wie hier Arbeit entlang von personalen Zuständigkeiten (diplomiertes Personal vs. Hilfskräfte) in einer berufsspezifischen Fachsprach lichkeit kompetent koordiniert wird.

Diese Fachsprache wurde in einem Prozess teilnehmender Beobachtung systematisch erschlossen und in Anlehnung an Spradley (1980, 140-154) dem kulturellen Thema „Ist eine Art Arbeit der Pflegenden für den/die Patientin“ zugefuihrt Dies wird in der Sprache des Feldes als ,direkte Pflege" bezeichnet, im iche Arbeiten, dic zwar nicht face-to-face mit zu versorgenden Menschen stattfinden, aber einer hospitalisierten Person dennoch zugerechnet werden können, erhielten wir so ein umfassendes und brauchbares Lexik ner Pflegearbeit in der berufseigenen Sprachlichkeit. Insgesamt konnen 13 Domänen der Arbeit in der Pflege rekonstruiert werden, die mit zwisomplementen verbunden sind und so eine sehr präzise Beschreibung der Arbeiten ergeben, die sich direkt auf zu versorgende Personen beziehen. Diese Domänen - ein Begriff zur kategorialen Taxonomisierung Anthropologie (vgl. D'Andrade. von Wissensbeständen innerhalb der kognitiven Anthropologie (vgl. D' Andrade, 1995) - kommt eine zentrale Stellung zu, da sie gewissermassen so etwas wie eine berufsgebundene Kurzstenographie der Arbeit abgeben (vgl. auch das Zitat von Lakoff weiter oben). In Organisationen, die rund um die Uhr und an 365 Tagen im Jahr aktiv sind und demnach eine ständige Personalrotation

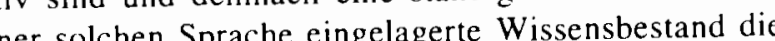
benötigen, ist der in einer solchen Sprache Voraussetzung für routinemässiges Funktionieren. In der Folge und in Anpassung an die Kultur, in der die Arbeiten stattfanden, haben wir Domänen als "Variablengruppen“ und die Komplemente als „Pflegevariablen“ bezeichnet

Die Konstruktionsarbeit des Variablenkatalogs erfolgte in zwei Schritten. Erstens mussten in einer umfassenden Liste die Begriffe für die im beruflichen Relevanzsystem bedeutsamen Tätigkeiten gefunden werden, und zweitens Relevanzsystem bedeutsamen Tatigkeiten gefunden werden, und zweitens mussten diesen Begriffen normative Zeitwerte zugeordnet werden. Es hat sich herausgestellt, dass der erste Schritt immer der kritischere war. In einem Gruppengespräch wird so unter Beteiligung eines Ethnographen (in diesem Kontext wissenschaftlicher Berater" genannt) versucht, eine semantische Struktur von Selbstbeschreibungen der Pflegearbeit herauszuarbeiten. Einzelne Tätigkeiten, die unter Umständen aus mehreren Teilschritten bestehen, bilden die unterste Ebene. Diese Tätigkeiten werden zu Variablengruppen zusammengefasst, und schliesslich bilden alle Gruppen zusammen die oberste Ebene der semantischen Struktur, nämlich der direkt einem Patienten zuordenbare Pflegeaufwand ${ }^{11}$

Bei der Auswahl der Begriffe zur Konstruktion des Tätigkeitslexikons für die Pflegenden geht es nicht nur darum, eine im Feld geläufige und verständliche Bezeichnung zu finden, sondern auch das Bedeutungsfeld des Begriffes auszuloten und in Form einer Beschreibung und mit Beispielen festzuhalten. Fällt dies relativ einfach, dann dürfte es sich um einen Wissensbestand aus der allgemeinen Pflegekultur handeln. Gelingt dies nicht auf Anhieb, so handelt es sich vermutlich um organisationsspezifische Wissensbestände, die in relativ langwierigen Diskussionen verhandelt werden müssen. Im ersten Fall lässt sich in der Regel problemlos ein Zeitwert zuordnen. Im zweiten Fall kann sich dies manchmal relativ schwierig gestalten und ein Begriff muss dann gewissermassen ausgehandelt werden. Es zeigt sich somit klar, dass der Variablenkatalog des Systems eine eigene Aushandlungsordnung (,, negotiated order") darstellt. ${ }^{12}$ Entscheidend ist dabei nun, dass diese Ordnung von Insidern der Pflege und nicht von Managementberatern ökonomischer Provenienz hergestellt wird. Das Ziel des Prozesses, die Rekonstruktion und Objektivierung der semantischen Struktur der wichtigsten Tätigkeitskategorien der pflegerischen Arbeit, kann nämlich nur von in diesem Berufsfeld sozialisierten Personen stimmig geleistet werden. Berufsfremde verwenden nach unseren Erfahrungen regelmässig und vorschnell analytische Kategorien aus der Arbeitswissenschaft, in denen die spezifischen Relevanzstrukturen der Handelnden im Feld übersehen werden.

Ein so gewonnener Variablenkatalog wird in einem nächsten Schritt in umfangreichen Validierungsstudien im Spital überprüft und revidiert, bevor er für die Ausprogrammierung an Computerfirmen freigegeben wird.$^{13}$ Modulare

11 Genau genommen werden nur die Tätigkeiten erfasst. dic erstens zeitlich ins Gewicht fallen (also messbar sind) und zweitens auch bezeichnet werden können.

12 Dieser Punkt kann nicht genug hervorgehoben werden; denn in naiver Sicht bilden diese Variablen „,die objektive Wirklichkeit" ab, doch aus sozial wissenschaftlicher Sicht wird diese gerade durch die Definition der Variablen hergestellt. In diesem Sinn sind auch andere Kategoriensysteme über Tätigkeiten - z. B. die Taxpunktkataloge der Ärzte - für sich genommen nicht objektiv, sondern sie erlangen ihre wirklichkeitskonstruierende Kraft erst durch die verhandelte Anerkennung darüber, dass sie eben objektiv seien.

13 Dabei werden quantitative und qualitative Verfahren kombiniert. Eine An der Validierung besteht darin, dass Pflegende das Lexikon der Tätigkeiten kommentieren und allenfalls ergänzen oder vereinfachen, und die zweite Art der Validierung geschieht mittels der Diskussion von Ergebnissen des Instruments, bezogen auf eine noch vorhandene und gut bekannte half"-Test eingesetzt. 
Erweiterungen spezifizieren besondere Kontexte, wie beispielsweise die Intensivstation oder die Psychiatrie. Auch sie verfügen über unterschiedliche. aber ebenfalls standardisierte Variablensets. In allen Fällen hat man sich auf ein Total von maximal 80 Tätigkeitsvariablen für jeden Handlungskontext der Pflege beschränkt. Diese Beschränkung ist zwar willkürlich, aber aufgrund von mittlerweile rund 10 Jahren Erfahrung mit diesem „Pflegelexikon“ wissen wir. dass mit 80 Variablen der Pflegeaufwand eines Patienten als Pflegearbeit genügend genau beschreibbar ist für den vorgesehenen Zweck der Objektivierung und Repräsentation von Pflegearbeit. Ausserdem gilt hier selbstverständlich auch eine Ökonomie der Daten, d. h. wenn mit mehr Messpunkten nur noch unwesentlich mehr verwertbare Information gewonnen wird, dann lohnt es sich nicht mehr genauer zu messen. Oder soziologischer formuliert, sind diese Kategorien insgesamt genügend mit den „folk theories" (Holland und Quinn. 1987, 7-21) der Pflegekultur gesättigt im Sinne der Idee der begründeten Theoriebildung (Glaser und Strauss. 1967). Das Instrument wird von den Pflegepersonen - unter Abzug der bereits erwähnten, nicht messbaren Dimensionen - denn auch als korrekte Beschreibung ihrer Arbeit anerkannt. Es wird in der organisatorischen Praxis vielerorts erfolgreich für die Steuerung und Optimierung der Pflegearbeit und auch für die Vertretung von Eigeninteressen des Berufs eingesetzt.

\section{Schlussfolgerungen}

Mittels ethnographischer Forschungsmethoden wurde ein Produkt hergestellt, dessen methodische und methodologische Fundierung im erzeugten Artefakt nicht mehr erkannt werden kann. Die Geertz'sche Charakterisierung der ethnographischen Arbeit von Evans-Pritchard als einer "Diaschau“ oder einer Ethnographie der Mittagszeit, in der hartes Licht mit wenig Schatten auf die dargestellten Dinge fällt, die auch als eine Art ,.anthropologisches Geometriebuch" (Geertz, 1993, 70) verstanden werden können, wurde hier noch zugespitzt: Die ethnographische Methode der Rekonstruktion von Wissensbeständen von Berufskulturen wurde dazu benützt, eine managerielle Wirklichkeitsrekonstruktionsmaschine herzustellen. Aufgrund von statistischen Objektivierungen der Arbeit vergangener Perioden können Entscheidungen für die Gestaltung der Arbeit in zukünftigen Zeiträumen legitimiert und durchgesetzt werden. Damit sind wir auch weit über die in der entsprechenden Literatur als ..applied ethnography" (Lyon, 1997, 3-27) gemachten Vorschläge zum Einsatz ethnographischer Kompetenz hinausgegangen. Wir haben nicht nur textförmige Beschreibungen im wissenschaftlichen Format zur Information und für die Reflexion der Praktiker über ihre Handlungsfelder hergestellt. sondern ihnen darüber hinaus Instrumente in die Hand gegeben, die ein veränderndes Eingreifen in das organisatorische Spiel der Aushandlung von Wirklichkeit ermöglichen.

Dennoch betrachten wir diese Arbeiten nicht als einen zusätzlichen Beitrag zu den Repräsentationsformen in der Ethnographie, denn diese durfte aus den dargelegten Gründen der dominanten Deutungskulturen im medizinischen Kontext ja gar nicht als solche in Erscheinung treten. Die Generierung der Beschreibungskategorien der Pflegearbeit und die funktionierende Einpassung in ein komplexes Organisations- und Professionsfeld ist aus unserer Sicht aber ausschliesslich dank dem ethnographischen Wissen und der zugehörigen verfremdenden Perspektive der Konstruktion sozialer Wirklichkeit gelungen. Nur wer damit ausgerüstet ist, kann als professioneller Fremder (Agar, 1996, 1-51) und Grenzgänger zwischen den Organisations- und Wissenschaftskulturen praktische Probleme der objektivierenden Darstellung von Arbeit lösen. Wir sind der Ansicht, dass bedenkenswerterweise gerade die ethnographische Orientierung, mit ihrer berechtigterweise grossen Distanz gegenüber Zahlen als Wirklichkeitsbeschreibungen, dieses Instrument stark und erfolgreich gemacht hat. ${ }^{14}$ Allein so ist es gelungen, in der in Frage stehenden Kultur des modernen Spitals eine anschlussfähige und passende kommunikative Form für die Darstellung von Arbeit in der Pflege - eine Art „Ethnostatistik“ - zu schaffen.

\section{LITERATURVERZEICHNIS}

Agar, Michael H. (1996), The Professional Stranger. An Informal Introduction to Ethnography. New York: Academic Press.

Assessment. Classification and Epidemiology Group (2000), ICIDH-2 Internationale Klassifikation der Funktionsfähigkeit und Behinderung. Beta-2 Entwurf. Genf: World Health Organization.

Becker. Howard S., Blanche Geer, Everett C. Huges und Anselm L. Strauss (1997), Boys in White. Student Culture in Medical School. New Brunswick/London: Transaction Publishers.

Berthou, Anne und Alain Junger (1999). NURSING data. Rapport intermédiaire. Lausanne: ISE.

D'Andrade. Roy (1995), The Development of Cognitive Anthropology, Cambridge: Cambridge University Press

Doenges. Marilynn E. und Moorhouse M.F. (1993), Pflegediagnosen und Massnalmen. Bern/ Göttingen/Toronto/Seattle: Huber.

Eberle, Thomas S. (1984). Sinnkonstitution in Alltag und Wissenschuft. Der Beitrag der Phänomenologie an die Methodologie der Sozialwissenschaften. Bern: Paul Haupt.

Eco, Unberto (1993), Das Foucault'sche Pendel. München: Deutscher Taschenbuchverlag.

Etzioni, Amitai (1969). The Semi-Professions and their Organization. Teachers, Nurses. Social Workers, New York: The Free Press.

14 Siehe dazu: Gephart (1988) mit seinen Ausführungen zu ..Ethnostatistics: Qualitative Foundations of Quantitative Reserach". 
Fineman. Stephen (1993), Organizations as Emotional Arenas, in: Stephen Fineman, Hrsg., Emotions in Organizations, London/Thousand Oaks/New Dehli: Sage, 9-35.

Finzen, Asmus (1999), Soziologie als Mutterdisziplin der Sozialpsychiatrie, in: Christoph Maeder Claudine Burton-Jeangros und Mary Haour-Knipe. Hrsg., Gesundheit. Medizin und Gesellschaft. Beiträge zur Soziologie der Gesundheit, Zürich: Seismo, 116-144.

Foucault, Michel (1988) Die Geburt der Klinik. Eine Archäologie des ärztlichen Blicks. Frankfur am Main: Fischer

Garrinkel. Harold und Harvey Sacks (1976), Über formale Strukturen praktischer Handlungen. in: Ethnomethodologie. Beiträge zu einer Soziologie des Alltagshandelns, Elmar Weingarten; Fritz Sack und Jim Schenkein, Hrsg., Frankfurt am Main: Suhrkamp. 130176.

Geertz. Clifford (1993), Die künstlichen Wilden, München/Wien: Fischer.

Gephart. Robert P. Jr. (1988), Ethnostatistics: Qualtiative Foundations for Quantitative Research, Newbury Park: Sage.

Glaser, Barney G. und Anselm L. Strauss (1967). The Discovery of Grounded Theory: Strategies for Qualitative Research, Chicago: Aldine.

Günter. Bernhard J. und Maeder Christoph (1994), Ein System zur Erfassung des Pflegeaufuandes. Darstellung der Methode SEP des Universitätsspitals in Zürich, Muri: Schriftenreihe der Darstellung der
SGGP Nr. 37.

Hall, Stuart (1997). Representation: Cultural Representations and Signifying Practices, London: Sage

Holland, Dorothy und Naomi Quinn. Hrsg. (1987). Cultural Models in Language and Thought. Cambridge: Cambridge University Press.

James, Allen E. (1996), Long Term Care Facility Resident Assessment Instrument. Users Manual. New York: Springer Publishing Company.

Kaplan, Robert S. und David P. Norton (1997), Balanced Scorecard. Strategien erfolgreich unsetzen. Stuttgart: Schäffer-Poeschel.

Lakoff, George (1990). Women. Fire, and Dangerous Things. What Categories Reveal about the Mind. Chicago/London: University of Chicago Press

Lyon, Eleanor (1997). Applying Ethnography. Journal of Comtemporary Ethnography. Special Issue: Applving Ethnographw. 1/26. 3-27.

Maeder, Christoph und Achim Brosziewski (1997). Ethnographische Semantik: Ein Weg zum Verstehen von Zugehörigkeit, in: Ronald Hitzler und Anne Honer. Hrsg.. Sozialwissenschaffliche Hermeneutik, Opladen: Leske + Budrich, 335-362.

Maeder, Christoph: Urs Brügger und Ulrich Bamert (1998), Beschreibung der Methode LEP: Anv'endungsbereich Intensivpflege. St. Gallen/Zürich: Kantonsspital St. Gallen und Universitätsspital Zürich

Maeder. Christoph: Urs Brügger und Uirich Bamen (1999a). Beschreibung der Methode LEP. Grundmodul Psychiatriebereich. St. Gallen/Zürich: Kantonsspital St. Gallen und Universitätsspital Zürich

Maeder. Christoph: Urs Brügger und Ulrich Bamert (1999b). Beschreibung der Methode LEP. Anuendungsbereich Gesundleits- und Krankenpflege für Erwachsene und Kinder im Spital. St. Gallen/Zürich: Kantonsspital St. Gallen und Universitätsspital Zürich.

McCloskey. Joanne C. und Gloria M. Bulechek (1996), Nursing Interventions Classifications (NIC). St. Louis/Boston/Baltimore usw.: Mosby

McNeal. Patricia: Carol Hutelmyer und Patrick Abrami (1988). Making in Work Right for You: Acuity Recording and Prolessional Nursing Care, Nursing Management, 10, 50-54. Russel-Hochschild. Arlie (1983), The Managed Heart. Commercialization of Human Feeling
Berkeley/Los Angeles/London: University of California Press.

Schneider, Gerald (1987). Interaktion auf der Intensivstation. Berlin: Michael-Ernst-Pörksen. Spradley, James P. (1979), The Ethnographic Interview, New York: Holt. Rinehart \& Winston. Spradley, James P. (1980), Participant Obsertation, New York: Holt, Rinehart \& Winston.

Strauss, Anselm; Leonard Schatzman; Rue Bucher. Danuta Ehrlich und Melvin Sabshin (1975), Negotiated Order and the Co-ordination of Work. in: Anselm Strauss, Hrsg. . Professions. Work and Careers, New Brunswick/New Jersey: Transaction Books. 175-202

Strauss. Anselm L. (1978), Negotiations. Varieties. Contexts, Processes, and Social Order, San Franzisco: Jossey-Bass.

Strauss. Anselm L.; Shizuko Fagerhaugh. Barbara Suczek und Carolyn Wiener (1997), Social Organization of Medical Work. New Brunswick//London: Transaction Publishers.

Strauss, Anselm L:; Leonard Schatzman; Danuta Ehrlich, Rue Bucher und Melvin Sabshin. (1963) The Hospital and It's Negotiated Order, in: Freidson, E., Hrsg.. The Hospital in Modern Socien: N. N. New York, 147-169.

Weber. Max ([1922] 1972), Wirtschaft und Gesellschaft: Grundriss der verstehenden Soziologie, Tübingen: J.C.B. Mohr, 5. Auflage.

Werner, Oswald und Mark G. Schoepfle (1987), Systematic Fieldwork. Ethnographic Analysis and Data Management. Newbury Park/London/New Dehli: Sage.

Werner. Oswald und Mark G. Schoepfle (1986), Systematic Fieldw'ork. Foundarions of Erhnography and Interviewing. Newbury Park/London/New Dehli: Sage

Anschrift des Autors:

Dr. Christoph Macder

Universität St. Gallen

Soziologisches Seminar

Tigerbergstr. 2

9000 St. Gallen

Tel.: $071 / 2242817$

www.sfs.unisg.ch

E-mail: Christoph.Maeder@unisg.ch 